

\title{
VEGFR2-targeted contrast-enhanced ultrasound to distinguish between two antiangiogenic treatments
}

Thomas Payen, Alexandre Dizeux, Capucine Baldini, Delphine Le

Guillou-Buffello, Michele Lamuraglia, Eva Comperat, Olivier Lucidarme, S. Lori Bridal

\section{To cite this version:}

Thomas Payen, Alexandre Dizeux, Capucine Baldini, Delphine Le Guillou-Buffello, Michele Lamuraglia, et al.. VEGFR2-targeted contrast-enhanced ultrasound to distinguish between two antiangiogenic treatments. Ultrasound in Medicine \& Biology, 2015, 41 (8), pp.2202-2211. 10.1016/j.ultrasmedbio.2015.04.010 . hal-01167020

\section{HAL Id: hal-01167020 \\ https://hal.sorbonne-universite.fr/hal-01167020}

Submitted on 23 Jun 2015

HAL is a multi-disciplinary open access archive for the deposit and dissemination of scientific research documents, whether they are published or not. The documents may come from teaching and research institutions in France or abroad, or from public or private research centers.
L'archive ouverte pluridisciplinaire HAL, est destinée au dépôt et à la diffusion de documents scientifiques de niveau recherche, publiés ou non, émanant des établissements d'enseignement et de recherche français ou étrangers, des laboratoires publics ou privés. 


\title{
VEGFR2-targeted contrast-enhanced ultrasound to distinguish between two antiangiogenic treatments
}

Thomas Payen, $\mathrm{PhD}^{1}$, Alexandre Dizeux, $\mathrm{MD}^{1}$, Capucine Baldini, $\mathrm{MD}^{1}$, Delphine Le Guillou-Buffello, $\mathrm{PhD}^{1}$, Michele Lamuraglia, $\mathrm{PhD}^{1,2}$, Eva Comperat, $\mathrm{PhD}^{3}$, Olivier Lucidarme, $\mathrm{PhD}^{4}$, S. Lori Bridal, $\mathrm{PhD}^{1}$

${ }^{1}$ Sorbonne Universités, UPMC Univ Paris 06, INSERM, CNRS, Laboratoire d'Imagerie Biomédicale, F75006, Paris, France

${ }^{2}$ Medical Oncology Department, Hopital Louis-Mourier, AP-HP, Colombes, France

${ }^{3}$ Anatomic Pathology Department, Pitié-Salpêtrière Hospital, AP-HP, Paris, France

${ }^{4}$ Sorbonne Universités, UPMC Univ Paris 06, INSERM, CNRS, AP-HP, Laboratoire d'Imagerie Biomédicale, F-75013, Paris, France

Corresponding author: Sharon Lori Bridal

\author{
Laboratoire d'Imagerie Biomédicale, UPMC, UMR 7371 - U1146 \\ 15 rue de l'Ecole de Médecine \\ 75006 Paris, France \\ Tel.: $\quad+33(0) 144419605$ \\ Fax. (+33) 146335673 \\ E-mail : lori.bridal@upmc.fr
}

The authors declare no conflict of interest.

\section{Abstract}


This study aims to evaluate the capacity of BR55, an ultrasound contrast agent specifically targeting the vascular endothelial growth factor receptor 2 (VEGFR2), to distinguish specific anti-VEGFR2 therapy effect with sunitinib from other antiangiogenic effects with a therapy (imatinib) that does not directly inhibit VEGFR2. Sunitinib, imatinib and placebo were administered daily for 11 days (264 hours) to 45 BalbC mice bearing ectopic CT26 murine colorectal carcinomas. During the course of therapy, B-mode, contrast-enhanced and VEGFR2-targeted contrast ultrasound was performed to assess the tumor morphology, the vascularization and the VEGFR2 expression, respectively. The angiogenic effects on these three aspects were characterized using the tumor volume, the contrast-enhanced area and the differential targeted enhancement (dTE). Necrosis, microvasculature and expression of VEGFR2 were also determined by histology and immunostaining. B-mode imaging demonstrated that tumor growth was significantly decreased in sunitinib-treated mice at Day $11(p<0.05)$ whereas imatinib did not impact growth. Functional evaluation showed that the contrast-enhanced area decreased significantly $(p<0.02)$ and by similar amounts under both antiangiogenic treatments by Day 8 (192 hours): - 23\% for imatinib and $-21 \%$ for sunitinib. No significant decrease was observed in the placebo group. Targeted-contrast imaging showed lower dTE, i.e. lower levels of VEGFR2 expression, in sunitinib-treated mice relative to placebo from 24 hours $(p<0.05)$ and relative to both placebo and imatinibtreated mice from 48 hours $(p<0.05)$. Histological assessment of tumors after the final imaging showed that necrotic area was significantly higher for the sunitinib group (21\%) compared to the placebo $(8 \%, p<0.001)$ and imatinib $(11 \%, p<0.05)$ groups. VEGFR2-targeted ultrasound was able to sensitively differentiate the anti-VEGFR2 
effect from reduced area of the tumor with functional flow that was produced by both antiangiogenic agents. BR55 molecular imaging was, thus, able to detect both early therapeutic response to sunitinib in CT26 tumors as soon as 24 hours after the beginning of the treatment, and also provided early discrimination (48 hours) between tumor response during antiangiogenic therapy targeting VEGFR2 expression and antiangiogenic therapy without direct action on this receptor.

Keywords: Ultrasound, targeted microbubbles, antiangiogenic treatment, VEGFR2 


\section{Introduction}

Antiangiogenic drugs have been developed to counter the imbalance between proand anti-angiogenic factors in tumors (Weis and Cheresh 2011). Many different signaling molecules and pathways influence the angiogenic response, and antiangiogenic drugs modify the interaction between tumor cells and their microenvironment. Tyrosine Kinase Inhibitors (TKI) are a specific antiangiogenic class inhibiting one or several angiogenic receptors and pathways. Imatinib is a tyrosine kinase inhibitor of SCFR (Stem Cell Growth Factor Receptor), BCR-ABL (Break Point Cluster-Abelson gene) and platelet-derived growth factor receptors (PDGFR) that targets pericytes and vascular smooth muscle cells (Capdeville et al. 2002). It is used in the treatment of patients with gastrointestinal stromal tumor (GIST) (Balachandran et al. 2011; McCarty et al. 2007). Sunitinib inhibits multiple TK receptors including PDGFR and VEGFR2 (also known as KDR, kinase insert domain protein receptor) (Faivre et al. 2007). In particular, this treatment is approved for treatment of imatinib-resistant GIST. Monitoring precise modifications of biomarkers such as VEGFR2 expression and modifications of the microvascular network during antiangiogenic therapy should help to rapidly identify patient responders and eventual signs of resistance, allowing personalized medicine.

Dynamic Contrast-Enhanced Ultrasound (DCE-US) is well suited for non-invasive and specific antiangiogenic monitoring (Lamuraglia et al. 2010; Leguerney et al. 2012). US contrast agents are encapsulated gas microbubbles with a size close to red blood cells which restricts their biodistribution to the vascular compartment. Due to their high echogenicity, these blood flow tracers can then be used for non-invasive, real-time 
assessment of the tumor vasculature and microvasculature. Targeted contrast agents, with the addition of ligands on the microbubble shell, can be used to bind to specific biological markers expressed in the vasculature for ultrasonic detection. BR55 is a VEGFR2-targeted microbubble developed by Bracco Suisse SA (Geneva, Switzerland). Studies have shown its specificity and capacity to assess VEGFR2 expression in vivo (BaronToaldo et al. 2014; Marinelli et al. 2014; Pochon et al. 2010; Tardy et al. 2010; Willmann et al. 2008) as well as its ability to monitor antiangiogenic treatments (BaronToaldo et al. 2014; Marinelli et al. 2014; Pysz et al. 2010). Marinelli et al. (Marinelli et al. 2014) and BaronToaldo et al. (BaronToaldo et al. 2014) monitored sorafenib therapy in hepatocelular carcinoma models using BR55 detecting early changes in VEGFR2 expression. Pysz et al. (Pysz et al. 2010) have demonstrated detection of the anti-VEGFR2 antibody blockade using BR55 in a human colon cancer xenograft model in mice. However, these longitudinal studies did not investigate whether the decreased BR55 signal observed resulted from specific anti-VEGFR2 effect or from the anti-vascular effect of these treatments (Liu et al. 2006; Pastuskovas et al. 2012) which would lead to reduced microbubble distribution and thus to lower targetedcontrast enhancement.

The ability to distinguish the different effects of antiangiogenic treatments has received little attention. The objective of this study was to assess the capacity of BR55 microbubbles to specifically detect the anti-VEGFR2 of sunitinib from that of imatinib, an antiangiogenic therapy that does not directly inhibit VEGFR2. Two TKI treatments were monitored: sunitinib (Sutent $\AA$, Pfizer, New York, NY) which targets multiple receptors including VEGFR2 and imatinib (Glivec®), Novartis, Basel, Switzerland) which does not 
have any direct action on this particular receptor. The capacity of BR55 to detect and distinguish the effects of these two drugs was studied in a mouse colorectal carcinoma model. 


\section{Materials and methods}

\section{Murine subcutaneous tumor model}

All experiments were approved by the French Ministry of Agriculture (protocol authorization: Ce5/2012/082). Mouse colorectal carcinoma CT26 (ATCC, Manassas, VA, USA) tumor fragments $\left(\sim 30 \mathrm{~mm}^{3}\right)$ were implanted subcutaneously in the right flank of 45, six-week-old female BalbC mice (Janvier, Le Genest-St-Isle, France).

\section{Therapy}

Therapy was started 11 days after fragment implantation when the tumors reached a volume of approximately $100 \mathrm{~mm}^{3}$. Mice were then randomized according to tumor size and perfusion in 3 groups of 15 animals: sunitinib, imatinib and placebo.

Imatinib mesylate (STI571) was freshly diluted in sterile PBS at $25 \mathrm{mg} / \mathrm{mL}$ and administered per os (orally) at $100 \mathrm{mg} / \mathrm{kg}$ twice a day ( 8 am and $8 \mathrm{pm}$ ) (Wolff et al. 2004) which corresponds to a volume of approximately $100 \mu \mathrm{L}$ for a $25-\mathrm{g}$ mouse.

Sunitinib (SU11248) was solubilized every 4 days at $10 \mathrm{mg} / \mathrm{mL}$ in $30 \%(\mathrm{v} / \mathrm{v})$ Cremophor EL (Sigma Aldrich), 30\% (v/v) polyethylene glycol 400, 10\% (v/v) ethanol, and $10 \%(\mathrm{wt} / \mathrm{v})$ glucose and was administered at $40 \mathrm{mg} / \mathrm{kg}$ once daily per os (Mendel et al. 2003) (about $100 \mu \mathrm{L}$ for a $25-\mathrm{g}$ mouse).

The mice in the placebo group received $100 \mu \mathrm{L}$ of PBS once daily per os.

\section{Contrast agents}

BR55 microbubbles (Bracco Spa, Milan, Italy) were used for the assessment of vascularization (Tardy et al. 2010) and VEGFR2 expression (Hyvelin et al. 2013). A 
lipopeptide showing strong and selective interaction with human and murine VEGFR2 is incorporated into the BR55 shell (Pillai et al. 2010; Shrivastava et al. 2005). Before use, the contrast agents are reconstituted with $2 \mathrm{~mL}$ of a $5 \%$ glucose solution resulting in a microbubble suspension containing $2 \times 10^{9} \mathrm{MB} / \mathrm{mL}$.

\section{In vivo imaging}

US imaging was performed using a Sequoia 512 US system (Acuson, Siemens, Moutain View, USA) with a $15 \mathrm{~L} 8 \mathrm{w}$ probe. The mechanical index was fixed at $\mathrm{Ml}=0.1$ to minimize microbubble destruction and the dynamic range was set at $80 \mathrm{~dB}$. The TGC was centered and the acoustic focus was placed at the center of the largest tumor cross section. The imaging was performed in fundamental brightness mode ( $\mathrm{B}$ mode) at 14 $\mathrm{MHz}$ for size assessment and manual delimitation of a region of interest following the tumor contours. Then, the transmit frequency was decreased to $7 \mathrm{MHz}$ and Cadence Contrast Pulse Sequencing (CPS) was used to detect the nonlinear signals from the microbubbles.

Injection was performed automatically using a syringe pump in a controlled system implemented in our laboratory based on a system developed by Bracco Suisse SA (Dizeux et al. 2012; Hyvelin et al. 2013). The microbubbles were injected in the tail vein at a dose of $1 \mathrm{~mL} / \mathrm{kg}$ to provide approximately $5 \times 10^{7} \mathrm{MBs}$ (Pysz et al. 2010). The imaging sequence consisted in a 1 minute bolus recorded at 1 frame/s followed by frame acquisition performed every 15 seconds for 10 minutes. Ten minutes after injection, the frame rate was increased to $1 \mathrm{fps}$ for 10 seconds. Then, a high-power acoustic flash was used to destroy the bubbles in the imaged-plane ( $\mathrm{Ml}=1.9$ for 2 
consecutive frames). After 10 seconds, data were then acquired for 10 seconds to assess re-circulating bubbles (Figure 1) (Hyvelin et al. 2013; Pochon et al. 2010; Tardy et al. 2010; Willmann et al. 2008). Throughout the imaging session, mice were kept on a thermostatically heated stage and anesthetized with $1.5 \%$ isoflurane in medical air at 1 L/min (Constantinides et al. 2011). The operator was blinded concerning the therapy received by the individual mice being imaged.

\section{Analysis}

In each group, nine mice from which imaging data were acquired at each time-point were used for therapy follow-up and the 6 other animals were euthanized at different time points for intermediate histological evaluation (Figure 2). Blind evaluation of the different parameters selected to monitor the therapy response were made prior to, at $6 \mathrm{~h}, 24 \mathrm{~h}, 48 \mathrm{~h}$ and 4,8 , and 11 days after the beginning of the treatment.

\section{Tumor volume}

Tumor dimensions were measured in the largest cross-section plane in the longitudinal and transverse axis. The volume was approximated using the ellipsoid formula

$$
V=\frac{\pi}{6} a b c
$$

where $a$ is the length, $b$ is the width and $c$ is the thickness. 


\section{US analysis}

Contrast images recorded as DICOM files were linearized to provide echo-power signals proportional to microbubble concentration within regions of interest (Payen et al. 2013) and processed using laboratory-based analysis approach for functional analysis. A prototype quantification software developed by Bracco Suisse SA, Geneva, Switzerland, was used for molecular imaging (Tranquart et al. 2012).

\section{Vascularization assessment}

Customized analysis of the DCE-US sequences was performed to extract the mean linear echo-power, from all the $3 \times 3$ pixels regions of analysis in the region of interest. $A$ voxel was considered to be enhanced when its maximal value over time reached 105 arbitrary units at any time during the acquired sequence. This threshold was empirically determined based on the intensity distribution histograms calculated from all the recorded sequences. The contrast-enhanced area of the whole tumor was thus estimated.

\section{Evaluation of VEGFR2 expression}

VEGFR2 expression was assessed using the late BR55 enhancement. Ten minutes after injection, the pre-destruction echo-power was calculated as the average echopower on 10 images acquired at $1 \mathrm{~Hz}$ just prior to the destruction. No detectable microbubble signal was observed in image frames acquired immediately after the flash, indicating effective destruction of targeted microbubbles. The circulating microbubbles were estimated 10 seconds after the flash as the mean signal on 10 images. The differential Targeted Enhancement (dTE) was then calculated as (signal pre- 
destruction) - (signal post-destruction) (Figure 1) (Hyvelin et al. 2013; Willmann et al. 2008). Due to the heterogeneous BR55 uptake, the dTE was only estimated in the area of the tumor that demonstrated microbubble uptake at any time during the time course of the acquisition according to the criteria described in Section 2.5.3.

\section{Histology}

Mice were euthanized and tumors were excised and frozen at different time points for histology (Figure 2). Tissue slices were incubated with either anti-mouse VEGFR2 antibody (CD309 (FLK1), Ebioscience, San Diego, CA, USA) in a 1:50 ratio (Zubair et al. 2010) or with monoclonal mouse anti-human CD31 antibody (Dakocytomation, Les Ulis, France) in a 1:40 ratio to stain endothelial cells (Giatromanolaki et al. 1997). Necrosis was also assessed using hematoxylin and eosin (H\&E) staining on the frozen slides.

The histological analysis was performed on two tumors per group for baseline (mice euthanized prior to therapy) and 10 tumors per group at the end of the study. Three mice from each group were euthanized between Day 0 and the end of the study for intermediate histological assessment, $\mathrm{N}=1$ per intermediate date (data not shown). The whole tumor slice, selected at the largest, central cross-section of the tumor, was evaluated under the microscope by an expert pathologist with magnifications from $\times 5$ to $x 40$. Three stained sections presenting the totality of the tumor were assessed for each tumor. Necrosis was estimated as a surface percentage based on estimations of the entire tumor cross-section and the necrotic cross-section made using a micrometric scale in the microscope objective. Both immunostainings were assessed qualitatively 
based on density and intensity with special focus on the spatial distribution between the tumor periphery and center.

\section{Statistics}

Statistics were performed using R software (version 3.0.1, http://www.r-project.org/). A Shapiro-Wilk test was used to check the populations were normally distributed. A global ANOVA test was performed to assess the overall difference before specific comparisons were made. Results are presented as the mean value \pm the standard deviation. A p-value of 0.05 or less was considered to indicate a significant difference.

All measurements (tumor volume, contrast-enhanced area and dTE) were normalized with respect to the baseline values (fixed at 100\%) for each mouse to evaluate individual variation. To assess the evolution over time, 95\% confidence intervals (95\% Cl) were calculated for each study date and each group. The parameter under investigation was considered to have significantly changed if the $95 \% \mathrm{Cl}$ did not include the $100 \%$ baseline value. A Mann-Whitney test was used to compare the results between the different groups at each study date.

The Mann Whitney test was also used to assess the significance of the differences in necrotic levels at the 264 hour time point. Wilcoxon signed-rank test was performed to compare the necrotic regions to the areas non-enhanced during microbubble passage. 


\section{Results}

\section{Tumor volume}

Before the beginning of treatment, the mean tumor volume was $124.5 \pm 20.9 \mathrm{~mm}^{3}$, $119.5 \pm 41.5 \mathrm{~mm}^{3}$ and $113.7 \pm 29.5 \mathrm{~mm}^{3}$ for the placebo, imatinib and sunitinib group, respectively. The evolution of the tumor size of each group is shown as the volume normalized to the baseline (fixed at 100\%) in the Figure $3 a$.

Imatinib did not impact the growth of the tumor during the study relative to placebo. Sunitinib significantly slowed $(p<0.05)$ the growth after 11 days $(264$ hours) of therapy (945.2 $\pm 185.5 \%$ for the sunitinib vs. $1484.8 \pm 163.8 \%$ for the placebo and $1388.8 \pm$ $179.2 \%$, for imatinib).

\section{Vascularization}

The tumor vascularization was monitored using the mean contrast-enhanced area. At baseline, $81.0 \pm 11.2 \%, 81.7 \pm 8.5 \%$, and $83.4 \pm 7.7 \%$ of the tumors were enhanced for the placebo, imatinib, and sunitinib group, respectively, showing no difference between the groups. The evolution of contrast-enhanced area with respect to these baseline values is shown for the three groups at each time point in Figure 3b.

The two treated groups showed a significant decrease $(p<0.05)$ of enhanced area after 8 days (192 hours) of treatment compared to their respective baseline. Normalized contrast-enhanced area dropped to $73.0 \pm 10.7 \%$ of the initial value for imatinib, and to $74.3 \pm 12.2 \%$ for sunitinib. Placebo mice demonstrated stable contrast-enhanced area until the end of the study. 
Significant intergroup differences appeared in contrast-enhanced area between the treated tumors and the placebo control after 8 days of therapy $(p<0.05)$. The trend was confirmed with the successive measurements at Day 11 . There was no significant difference between matched-day enhanced area in tumors treated with imatinib and sunitinib.

\section{VEGFR2 expression}

The VEGFR2 expression was monitored via the dTE parameter at each study point (Figure 3c). Before the beginning of treatment, there was no significant difference in dTE between the groups: $4864.6 \pm 3182.1$ a.u. for the placebo, $5069.7 \pm 3444.0$ a.u. for the imatinib, and $4807.8 \pm 2162.9$ a.u. for the sunitinib group.

The placebo and imatinib-treated tumors did not show any significant intragroup evolution in the dTE variation. On the contrary, sunitinib-treated tumors demonstrated a significant decrease from the 24 hour point $(77.4 \pm 21.2 \%, p<0.05)$ until the end of the study.

The dTE variation in imatinib-treated tumors was not significantly different from the placebo group at any study dates. However, this parameter was able to detect the effects of sunitinib from the 24 hour point $(105.4 \pm 30.2 \%$ for the placebo group, $p<$ 0.05). Moreover, the two treatments could be distinguished after 48 hours of therapy $(32.0 \pm 11.4$ and $70.6 \pm 30.4 \%$ for the sunitinib and imatinib group, respectively, $p<$ 0.05), and the relatively lower level of dTE in the sunitinib group was sustained throughout the study.

\section{Histology}




\section{Hematoxylin and eosin (H\&E) staining}

H\&E staining results are summarized in Figure 4. Representative images are shown in Figure 5.

The mean necrotic area increased significantly $(p<0.05)$ for the three groups between baseline and the end of the treatment period. There was no significant difference between the imatinib $(11.4 \pm 5.6 \%)$ and placebo $(7.5 \pm 3.8 \%)$ groups at the end of the study $(p>0.05)$. However, sunitinib-treated tumors demonstrated a significantly higher necrotic percentage $(20.8 \pm 7.4 \%)$ than the other two groups after 11 days (264 hours) of treatment $(p<0.05)$.

Moreover, for the three groups, the necrotic fraction was significantly lower than the tumor fraction without any BR55 microbubble uptake which corresponds to [100 (contrast-enhanced area)] $(\mathrm{p}<0.005)$.

\section{Immunostaining}

Representative images of CD31 and VEGFR2 staining are shown in Figure 6.

The immunohistological results did not allow the detection of any progressive evolution of staining over the study duration in any group. However, functional and molecular differences were qualitatively observed between groups at the end of the experiment. At Day 11 (264 hours), large vessels were identified using the CD31 staining in 8 out of the 10 placebo tumors and in 6 out of 10 imatinib-treated tumors whereas this feature did not appear in the sunitinib group. These vessels were mostly located in the peripheral tumor zone and CD309 staining detected VEGFR2 expression on their surface. Sunitinib-treated tumors presented small stained spots in both CD31 and CD309 staining with a higher vascular density in the peripheral zone compared to the center. 


\section{Discussion}

This work shows that anti-VEGFR2 effect can be discriminated from reduced vascularization without anti-VEGFR2 action using BR55 in the murine CT26 tumor model. The morphological, functional and molecular effects of sunitinib which inhibits VEGFR2 and imatinib without any direct action on this particular receptor were monitored for 11 days. The distinction of the anti-VEGFR2 effect from the anti-vascular action was possible using BR55 microbubbles for VEGFR2 expression imaging as soon as two days after the initiation of therapy.

Sunitinib and imatinib did not have the same impact on lesion volume development. Significant differences appeared after 11 days (264 hours) of therapy when sunitinib was shown to significantly reduce tumor growth whereas imatinib did not demonstrate any effect on tumor size compared to the control group. Volumetric reduction of CT26 tumors treated with sunitinib has been observed in other colorectal tumor models. Mendel et al. (Mendel et al. 2003) monitored the effect of sunitinib at the same dose in HT29 and Colo205 cancer models for 101 days. They observed tumor volume regression after $74(-62 \%)$ and $35(-13 \%)$ days for each model. Moreover, the lack of growth reduction in colorectal tumors treated with imatinib has previously been observed in human cancer models such as HT29 (McCarty et al. 2007).

Many studies have shown that size evaluation may not detect the effects of angiogenic drugs which impact the vasculature of the tumor (Leach et al. 2005) while functional assessment enables detection of antiangiogenic response that can precede volume reduction. Assessment of the spatial distribution of functional flow with contrastenhanced area enabled the detection of the impact of both drugs on microvascular 
distribution after 8 days (192 hours) of therapy. Sunitnib's effect on vascularization has previously been reported in several studies. Osusky et al (Osusky et al. 2004) observed an inhibition of the formation of new vessels in Lewis Lung carcinoma, and microvascular density reduction with sunitnib treatment was detected in tumor types with various molecular expressions including HT-29 and Colo205 human colon carcinoma (Potapova et al. 2006). In this work, imatinib had no effect on tumor volume but did decrease the effective microvascularization which suggests that its main target, PDGFR, is expressed in the CT26 vasculature. PDGFR inhibition results in a lower vessel coverage by pericytes and a less functional vascularization (Kano et al. 2009).

Molecular imaging with BR55 demonstrated two interesting features. First, it allowed the earliest and most sustained detection of sunitinib's antiangiogenic effects. The dTE variation of the sunitnib-treated tumors was consistently lower than the placebo group from 24 hours after the beginning of the treatment to the end of the study. Pysz et al (Pysz et al. 2010) have shown that BR55 signal was significantly reduced (mean, 3.9fold $\pm 1.2 ; p=.03$ ) after administration of a VEGFR2-blocking antibody. This result suggests that BR55 signal specifically reflects VEGFR2 expression in vivo. Therefore sunitinib seems to impact VEGFR2 expression in CT26 as previously reported in other models such as mammary tumor models in the rat (Levashova et al. 2010). Other studies have shown that VEGFR2 inhibition may result in the internalization of this receptor which would then be inactive and inaccessible to intravascular tracers such as BR55 (Ewan et al. 2006). Secondly, this imaging technique was able to distinguish sunitinib action from imatinib effects after 2 days of therapy, whereas, evaluation of the contrast-enhanced area only detected the overall, and apparently similar, vascular 
alteration effect of these two angiogenic drugs developing at a later phase (at 192 hours). BR55 targeted imaging was able to specifically assess early anti-VEGFR2 effects (at 48 hours). Even though the PDGFR receptor targeted by imatinib is linked to the VEGF pathway (Hicklin and Ellis 2005), at the dose administered, this drug does not seem to significantly decrease VEGFR2 expression in the CT26 model. Such capacity to specifically evaluate drug effect on an individual axis of the angiogenic pathway could be very useful for evaluating mechanisms linked to therapeutic response and drug resistance in individual tumors.

In this study, BR55 targeted agent uptake was used for functional imaging as well as molecular imaging. Tardy et al. (Tardy et al. 2010) compared BR55 and SonoVue uptake in a Dunning rat tumor model. They observed that the wash-in phase of the microbubble uptake was similar for targeted and non-targeted agents in their model. At later stages of anti-angiogenic therapy in our model, comparison of echo-power vs. time curves from BR55 and Sonovue microbubbles made in a separate group of mice (data not shown) indicated that the BR55-targeting could perturb the kinetics of the microbubble flow even during the wash-in phase, in some cases. Therefore, in this study, the contrast-enhanced area was preferred over semi-quantification methods such as those proposed by Wei et al. (Wei et al. 1998) for assessment of tumor vascularization because it reflects microbubble spatial accessibility with no regard to flow kinetics. Because SonoVue and BR55 have similar size distributions (mean diameters of 1.8 and $1.5 \mu \mathrm{m}$, respectively (Hyvelin et al. 2013)), these agents should reach the same tumor regions. Although the contrast-enhanced area does not reflect precise features such as flow kinetics, the spatial distribution of the contrast 
enhancement has been shown to be related to the heterogeneity of the microvascular distribution which has been linked to tumor malignancy in previous studies (Agrawal et al. 2009; Junttila and de Sauvage 2013). The choice of the threshold value was empirical and it depends on different factors such as noise level, dynamic range and contrast-detection mode.

The distribution of microbubbles in the tumor was very heterogeneous in the CT26 tumors, especially at late stages. During the bolus passage, certain parts of the tumor were highly enhanced, while bubbles were not detected in other regions (Figure 1). The dTE reflecting the VEGFR2 expression was then evaluated in the tumor area where BR55 microbubbles demonstrated detectable presence during the time course of the 10-minute contrast sequence. Thus, only the fraction of tumor that microbubbles could reach and subsequently fix within, were assessed. In current contrast ultrasound methodology, functional and molecular parameters are typically evaluated as spatial means across the whole tumor. However, solid tumor vascularization is often very heterogeneous (Gillies et al. 1999; Jain 1988) affecting relative, local microbubble uptake. Inclusion of unperfused pixels in ROIs used for evaluation of targeted contrast assessment artificially lowers the mean dTE and can also bias estimates of functional parameters assessed with non-targeted contrast agents. Mean values may, therefore, be less sensitive to therapeutic response. Targeted and functional evaluation with ultrasound contrast agents, should, therefore, be estimated only in regions of the tumor accessible to microbubbles. By including analysis of parameters such as the contrastenhanced area, a more complete view of overall modifications in the microvascular distribution and changes in the flow and expression within the vascularized regions was 
developed. Care was taken in this work to reposition the imaging plane on the largest, central cross section of the tumor during follow-up. Analysis was, however, limited to a single, central plane of the tumor. Three-dimensional DCE-US are currently emerging (Sridharan et al. 2013) and should further empower the use of this modality for longitudinal evaluation of heterogenous tumor vascularization in the future by providing a volumetric assessment of parameters such as the contrast-enhanced volume.

Anesthesia with isoflurane can decrease heart rate $(H R)$, mean arterial pressure (MAP) and lead to other physiological effects. For the $1.5 \%$ isofluane level and anethesia durations used throughout this work relatively stable HR and MAP should be obtained (Constantinides et al. 2011).

The specific features of the vasculature structure and/or the blood flow which prevented the agent from entering central zones of these CT26 tumors at certain stages of their growth remains unclear. Often, regions without contrast enhancement in DCEUS are assimiliated with necrosis. However, the analysis of H\&E staining slides showed that the necrotic fraction was significantly lower than the tumor fraction without any microbubble enhancement for the three groups. Vascular structure (vessel density, diameter, tortuosity) may also explain the lack of agent detection in these regions. Immunostaining indicated a lower microvascular density with fewer large vessels in the sunitinib-treated tumors. However, these trends were not detected in the imatinib group which demonstrated a similar level of \% contrast-enhanced area in DCE-US. Further investigation of the mechanisms explaining the presence of regions without contrast enhancement in this model could be valuable for interpretation of contrast ultrasound in 
complex tumor environments. Moreover, 3D evaluation would provide useful information to understand the flow dynamics in tumor vascular trees.

To our knowledge, this study assessed for the first time the specific detection of antiVEGFR2 action of antiangiogenic therapy using BR55 in the murine CT26 tumor model. Sunitinib which targets VEGFR2 and imatinib which does not have any direct action on this particular receptor were used and their morphological, functional and molecular effects were monitored. The distinction of the effects of these two drugs was possible using BR55 microbubbles for VEGFR2 expression imaging after two days of therapy. These results open interesting perspectives for sensitive monitoring of microvascular and molecular modifications during antiangiogenic therapy to better reveal individual response and understand mechanisms behind therapeutic effectiveness. 


\section{Acknowledgements}

The authors thank Bracco Suisse SA for kindly providing the BR55, and SonoVue contrast agents and the prototype software for this study. We are also grateful to I. Tardy, J.M. Hyvelin, and F. Tranquart (Bracco Suisse SA) for helpful advice and discussion. 


\section{References}

Agrawal G, Su M-Y, Nalcioglu O, Feig SA, Chen J-H. Significance of breast lesion descriptors in the ACR BI-RADS MRI lexicon. Cancer 2009;115:1363-80.

Balachandran VP, Cavnar MJ, Zeng S, Bamboat ZM, Ocuin LM, Obaid H, Sorenson EC, Popow R, Ariyan C, Rossi F, Besmer P, Guo T, Antonescu CR, Taguchi T, Yuan J, Wolchok JD, Allison JP, DeMatteo RP. Imatinib potentiates antitumor T cell responses in gastrointestinal stromal tumor through the inhibition of Ido. Nat Med $2011 ; 17: 1094-100$.

BaronToaldo M, Salvatore V, Marinelli S, Palamà C, Milazzo M, Croci L, Venerandi L, Cipone M, Bolondi L, Piscaglia F. Use of VEGFR-2 Targeted Ultrasound Contrast Agent for the Early Evaluation of Response to Sorafenib in a Mouse Model of Hepatocellular Carcinoma. Mol Imaging Biol 2014;

Capdeville R, Buchdunger E, Zimmermann J, Matter A. Glivec (STI571, imatinib), a rationally developed, targeted anticancer drug. Nat Rev Drug Discov 2002;1:493502.

Constantinides $\mathrm{C}$, Mean R, Janssen BJ. Effects of isoflurane anesthesia on the cardiovascular function of the C57BL/6 mouse. ILAR J 2011;52:e21-e31.

Dizeux A, Payen T, Lechuga G, Lori Bridal S. Implementation of a controlled injection system for dynamic contrast-enhanced ultrasonography. 2012 IEEE Int Ultrason Symp leee, 2012;1:1-4.

Ewan LC, Jopling HM, Jia H, Mittar S, Bagherzadeh A, Howell GJ, Walker JH, Zachary IC, Ponnambalam S. Intrinsic Tyrosine Kinase Activity is Required for Vascular Endothelial Growth Factor Receptor 2 Ubiquitination, Sorting and Degradation in Endothelial Cells. Traffic 2006;7:1270-1282.

Faivre S, Demetri G, Sargent W, Raymond E. Molecular basis for sunitinib efficacy and future clinical development. Nat Rev Drug Discov 2007;6:734-45.

Giatromanolaki A, Koukourakis MI, Theodossiou D, Barbatis K, O’Byrne K, Harris AL, Gatter KC. Comparative evaluation of angiogenesis assessment with anti-factorVIII and anti-CD31 immunostaining in non-small cell lung cancer. Clin Cancer Res 1997;3:2485-92.

Gillies RJ, Schornack PA, Secomb TW, Raghunand N. Causes and effects of heterogeneous perfusion in tumors. Neoplasia 1999;1:197-207.

Hicklin DJ, Ellis L. Role of the vascular endothelial growth factor pathway in tumor growth and angiogenesis. J Clin Oncol 2005;23:1011-1027. 
Hyvelin J, Tardy I, Arbogast C, Costa M, Emmel P, Helbert A, Theraulaz M, Nunn A, Tranquart F. Use of Ultrasound Contrast Agent Microbubbles in Preclinical Research. Invest Radiol 2013;48:570-583.

Jain RK. Determinants of tumor blood flow: a review. Cancer Res 1988;48:2641-2658.

Junttila MR, de Sauvage FJ. Influence of tumour micro-environment heterogeneity on therapeutic response. Nature 2013;501:346-354.

Kano MR, Komuta Y, Iwata C, Oka M, Shirai Y, Morishita Y, Ouchi Y, Kataoka K, Miyazono K. Comparison of the effects of the kinase inhibitors imatinib, sorafenib, and transforming growth factor-beta receptor inhibitor on extravasation of nanoparticles from neovasculature. Cancer Sci 2009;100:173-80.

Lamuraglia M, Bridal SL, Santin M, Izzi G, Rixe O, Paradiso A, Lucidarme O. Clinical relevance of contrast-enhanced ultrasound in monitoring anti-angiogenic therapy of cancer: current status and perspectives. Crit Rev Oncol Hematol 2010;73:202-12.

Leach MO, Brindle KM, Evelhoch JL, Griffiths JR, Horsman MR, Jackson A, Jayson GC, Judson IR, Knopp M V, Maxwell RJ, McIntyre D, Padhani AR, Price P, Rathbone R, Rustin GJ, Tofts PS, Tozer GM, Vennart W, Waterton JC, Williams SR, Workman $P$. The assessment of antiangiogenic and antivascular therapies in early-stage clinical trials using magnetic resonance imaging: issues and recommendations. $\mathrm{Br}$ J Cancer 2005;92:1599-610.

Leguerney I, Lassau N, Koscielny S, Rodrigues M, Massard C, Rouffiac V, Benatsou B, Thalmensi J, Bawa O, Opolon P, Peronneau P, Roche A. Combining functional imaging and interstitial pressure measurements to evaluate two anti-angiogenic treatments. Invest New Drugs 2012;30:144-56.

Levashova Z, Backer M, Hamby C V, Pizzonia J, Backer JM, Blankenberg FG. Molecular imaging of changes in the prevalence of vascular endothelial growth factor receptor in sunitinib-treated murine mammary tumors. J Nucl Med 2010;51:959-66.

Liu L, Cao Y, Chen C, Zhang X, McNabola A, Wilkie D, Wilhelm S, Lynch M, Carter C. Sorafenib blocks the RAF/MEK/ERK pathway, inhibits tumor angiogenesis, and induces tumor cell apoptosis in hepatocellular carcinoma model PLC/PRF/5. Cancer Res 2006;66:11851-8.

Marinelli S, Salvatore V, Baron Toaldo M, Milazzo M, Croci L, Venerandi L, Pecorelli A, Palamà $\mathrm{C}$, Diana $\mathrm{A}$, Bolondi L, Piscaglia $\mathrm{F}$. Evaluation of the impact of transient interruption of antiangiogenic treatment using ultrasound-based techniques in a murine model of hepatocellular carcinoma. BMC Cancer 2014;14:403. 
McCarty MF, Somcio RJ, Stoeltzing O, Wey J, Fan F, Liu W, Bucana C, Ellis LM. Overexpression of PDGF-BB decreases colorectal and pancreatic cancer growth by increasing tumor pericyte content. J Clin Invest 2007;117:2114-2122.

Mendel D, Laird A, Xin X, Louie S, Christensen J, Li G, Schreck R, Abrams T, Ngai T, Lee L, Murray L, Carver J, Chan E, Moss K, Haznedar J, Sukbuntherng J, Blake R, Sun L, Tang C, Miller T, Shirazian S, McMahon G, Cherrington J. In vivo antitumor activity of SU11248, a novel Kinase Inhibitor Targeting Vascular Endothelial Growth Factor and Platelet-derived Growth Factor Receptors Determination of a Pharmacokinetic/Pharmacodynamic. Clin Cancer Res 2003;9:327-337.

Osusky KL, Hallahan DE, Fu A, Ye F, Shyr Y, Geng L. The receptor tyrosine kinase inhibitor SU11248 impedes endothelial cell migration, tubule formation, and blood vessel formation in vivo, but has little effect on existing tumor vessels.

Angiogenesis 2004;7:225-33.

Pastuskovas C V, Mundo EE, Williams SP, Nayak TK, Ho J, Ulufatu S, Clark S, Ross S, Cheng E, Parsons-Reponte K, Cain G, Van Hoy M, Majidy N, Bheddah S, dela Cruz Chuh J, Kozak KR, Lewin-Koh N, Nauka P, Bumbaca D, Sliwkowski M, Tibbitts J, Theil F-P, Fielder PJ, Khawli LA, Boswell CA. Effects of anti-VEGF on pharmacokinetics, biodistribution, and tumor penetration of trastuzumab in a preclinical breast cancer model. Mol Cancer Ther 2012;11:752-62.

Payen T, Coron A, Lamuraglia M, Le Guillou-Buffello D, Gaud E, Arditi M, Lucidarme O, Bridal SL. Echo-power estimation from log-compressed video data in dynamic contrast-enhanced ultrasound imaging. Ultrasound Med Biol 2013;39:1826-37.

Pillai R, Marinelli ER, Fan H, Nanjappan P, Song B, von Wronski MA, Cherkaoui S, Tardy I, Pochon S, Schneider M, Nunn AD, Swenson RE. A PhospholipidPEG2000 Conjugate of a Vascular Endothelial Growth Factor Receptor 2 (VEGFR2)-Targeting Heterodimer Peptide for Contrast-Enhanced Ultrasound Imaging of Angiogenesis. Bioconjug Chem 2010;2:556-562.

Pochon S, Tardy I, Bussat P, Bettinger T, Brochot J, von Wronski M, Passantino L, Schneider M. BR55: a lipopeptide-based VEGFR2-targeted ultrasound contrast agent for molecular imaging of angiogenesis. Invest Radiol 2010;45:89-95.

Potapova O, Laird AD, Nannini MA, Barone A, Li G, Moss KG, Cherrington JM, Mendel DB. Contribution of individual targets to the antitumor efficacy of the multitargeted receptor tyrosine kinase inhibitor SU11248. Mol Cancer Ther 2006;5:1280-9.

Pysz MA, Foygel K, Rosenberg J, Gambhir SS, Schneider M, Willmann JK. Antiangiogenic Cancer Therapy: Monitoring with Molecular US and a Clinically Translatable Contrast Agent (BR55). Radiology 2010;256:519-527. 
Shrivastava A, von Wronski MA, Sato AK, Dransfield DT, Sexton D, Bogdan N, Pillai R, Nanjappan P, Song B, Marinelli E, DeOliveira D, Luneau C, Devlin M, Muruganandam A, Abujoub A, Connelly G, Wu QL, Conley G, Chang Q, Tweedle MF, Ladner RC, Swenson RE, Nunn AD. A distinct strategy to generate high-affinity peptide binders to receptor tyrosine kinases. Protein Eng Des Sel 2005;18:417-24.

Sridharan A, Eisenbrey JR, Liu J-B, Machado P, Halldorsdottir VG, Dave JK, Zhao H, He Y, Park S, Dianis S, Wallace K, Thomenius KE, Forsberg F. Perfusion Estimation Using Contrast-Enhanced 3-dimensional Subharmonic Ultrasound Imaging: An In Vivo Study. Invest Radiol 2013;48:654-60.

Tardy I, Pochon S, Theraulaz M, Emmel P, Passantino L, Tranquart F, Schneider M. Ultrasound molecular imaging of VEGFR2 in a rat prostate tumor model using BR55. Invest Radiol 2010;45:573-8.

Tranquart F, Mercier L, Frinking P, Gaud E, Arditi M. Perfusion Quantification in Contrast-Enhanced Ultrasound (CEUS)-Ready for Research Projects and Routine Clinical Use. Ultraschall der Medizin 2012;33:S31-S38.

Wei K, Jayaweera AR, Firoozan S, Linka A, Skyba DM, Kaul S. Quantification of myocardial blood flow with ultrasound-induced destruction of microbubbles administered as a constant venous infusion. Circulation 1998;97:473-83.

Weis SM, Cheresh DA. Tumor angiogenesis: molecular pathways and therapeutic targets. Nat Med 2011;17:1359-70.

Willmann JK, Paulmurugan R, Chen K, Gheysens O, Rodriguez-Porcel M, Lutz AM, Chen IY, Chen X, Gambhir SS. US Imaging of Tumor Angiogenesis with Microbubbles Targeted to Vascular Endothelial Growth Factor Receptor Type 2 in Mice1. Radiology 2008;246:508-18.

Wolff NC, Randle DE, Egorin MJ, Minna JD, Ilaria RL. Imatinib mesylate efficiently achieves therapeutic intratumor concentrations in vivo but has limited activity in a xenograft model of small cell lung cancer. Clin Cancer Res 2004;10:3528-34.

Zubair AC, Malik S, Paulsen A, Ishikawa M, McCoy C, Adams PX, Amrani D, Costa M. Evaluation of mobilized peripheral blood CD34(+) cells from patients with severe coronary artery disease as a source of endothelial progenitor cells. Cytotherapy Elsevier, 2010;12:178-89. 


\section{Figure legends}

Figure 1: Diagram of the contrast sequence used in this BR55 study. The time-intensity curve shows the bolus passage with a peak 30 seconds after the microbubble injection, then the signal slowly decays. The agents are destroyed 10'30 after the injection and the flowing microbubbles are imaged for 30s. Mean signals are calculated on 10 seconds before and 10 seconds after destruction in the enhanced area. The difference in these mean echo-power (dTE) is assessed to evaluate the echo-power from bound microbubbles. CPS images show the signal enhancement at different time points with the tumor enhanced area outlined. CT26 tumor, 22 days after fragment implantation. The tumor is delimitated and the dark region outlined inside the tumor designates the non-enhanced area.

Figure 2: Timeline of the antiangiogenic treatment schedule and longitudinal tumor evaluation using targeted contrast ultrasound and immunostaining to assess tumor volume, vascularization and VEGFR2 expression.

Figure 3: Boxplot diagrams of the tumor volume (a), the contrast-enhanced area during the initial 60s passage of BR55 (b), and the dTE (c) all normalized with respect to baseline values (fixed at 100\%) (Significance of differences between groups at each measurement point: $\left.{ }^{*} p<0.05,{ }^{* *} p<0.01,{ }^{* *} p<0.001\right)$. 
Figure 4: Necrotic area evaluated on H\&E-stained slices at different time points for the three groups. Sections were obtained from 2 tumors at baseline, and 10 at 264 hours for each group (Significance of differences between groups at 264 hours: ${ }^{*} p<0.05$, $\left.{ }^{*} p<0.01,{ }^{* * *} p<0.001\right)$.

Figure 5: H\&E staining results for the imatinib (left), placebo (center, and sunitinib (right) groups at the end of the study. Necrosis (indicated by arrows) are observed in the imatinib and sunitinib groups.

Figure 6: Immunostaining of endothelial cells (CD31, left) and VEGFR2 (CD309, right). Representative microscopic (x10) images are shown from slides of placebo (top), imatinib (middle) and sunitinib-treated tumors (bottom) at 264 hours. The location of the field of view was selected to be toward the peripheral zone where imaging assessment demonstrated microbubble uptake and is indicated in a small image of the full section in the right bottom corner of each image. Large vessels (indicated by arrows) were not observed in sunitinib-treated tumors $(\mathrm{N}=10)$, whereas they were present in the placebo group. 


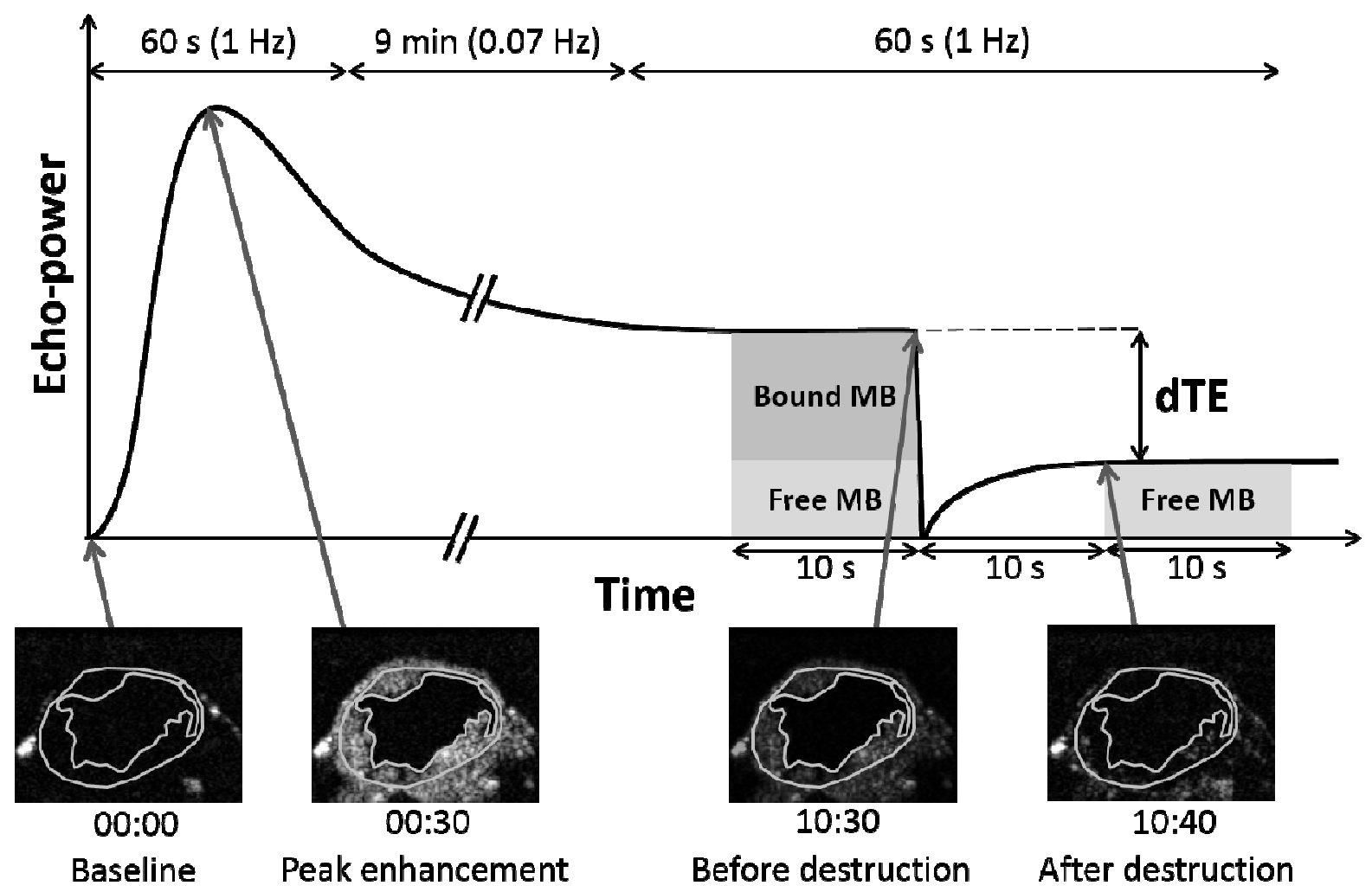

Figure 1 


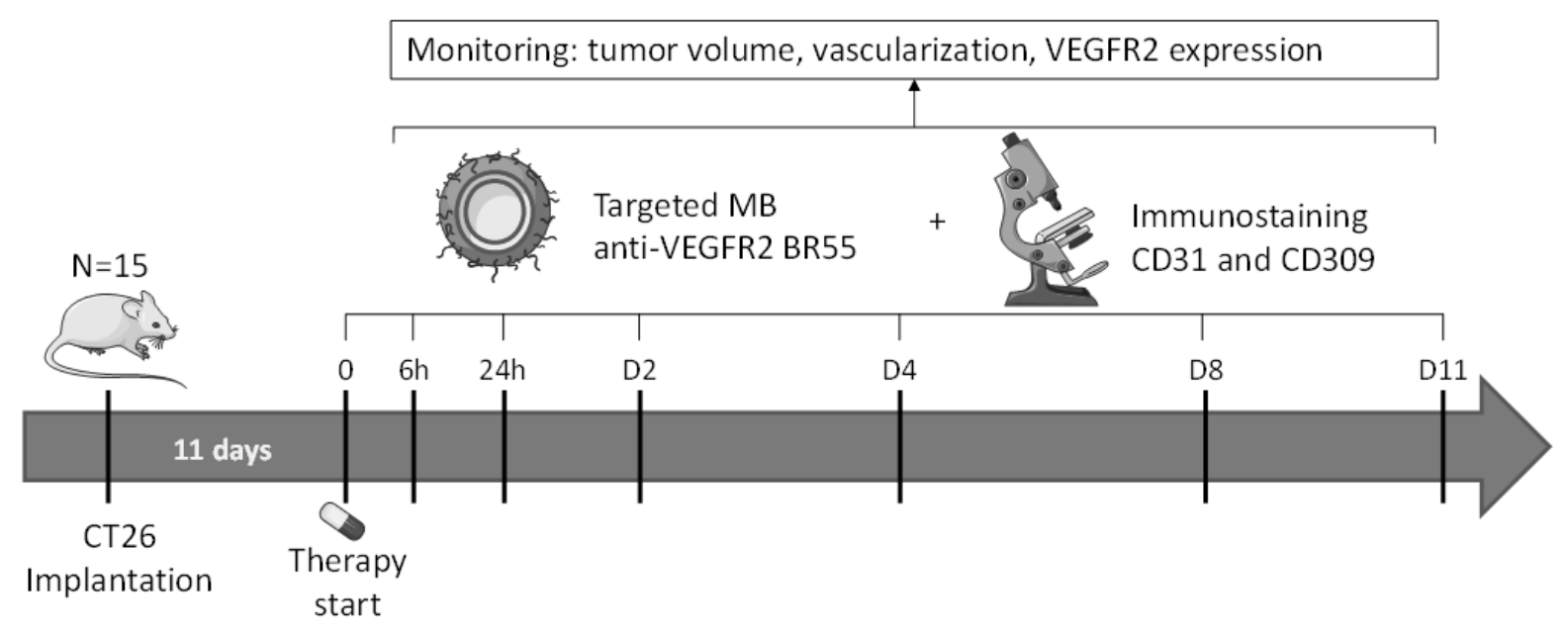

Figure 2 

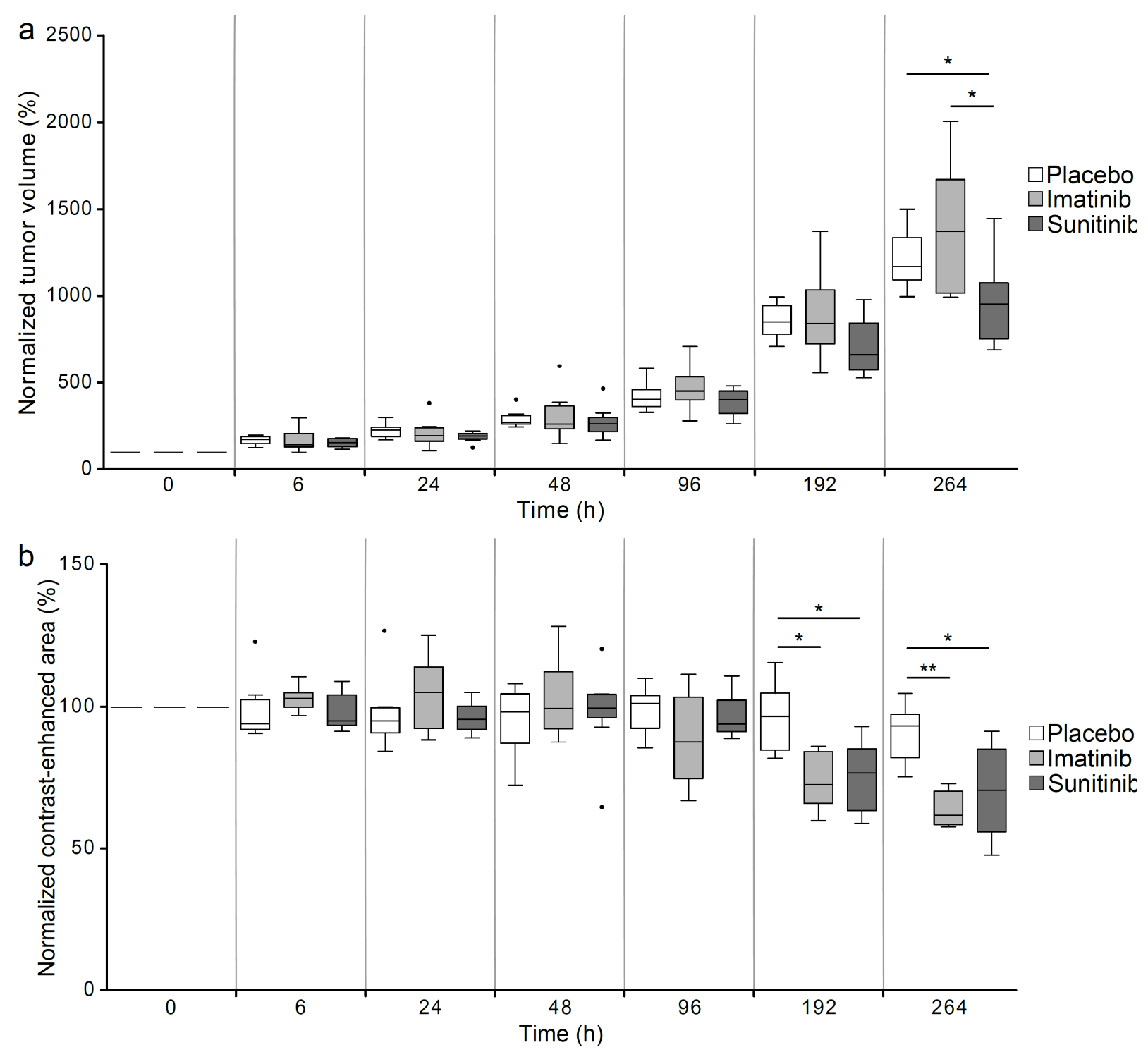

Figure 3 


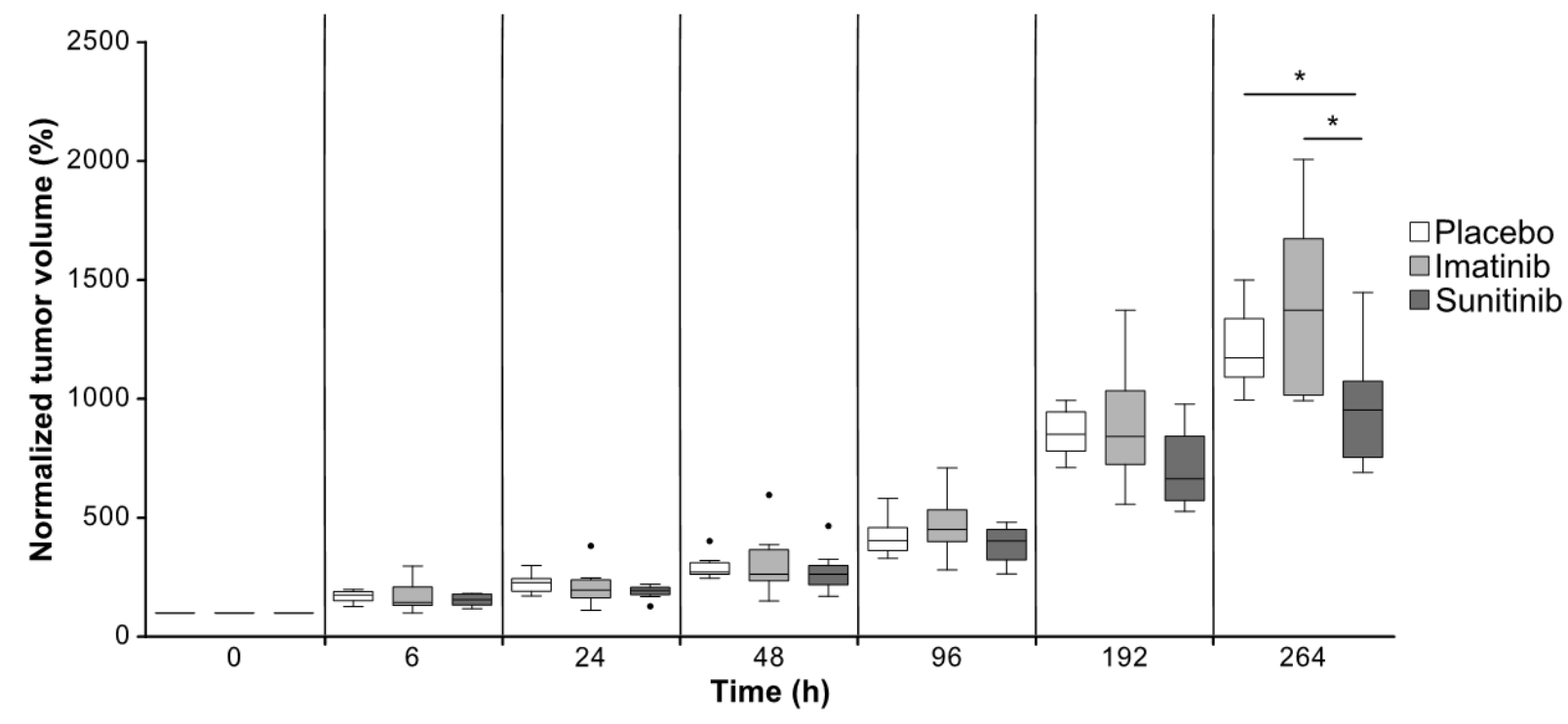

Figure 4

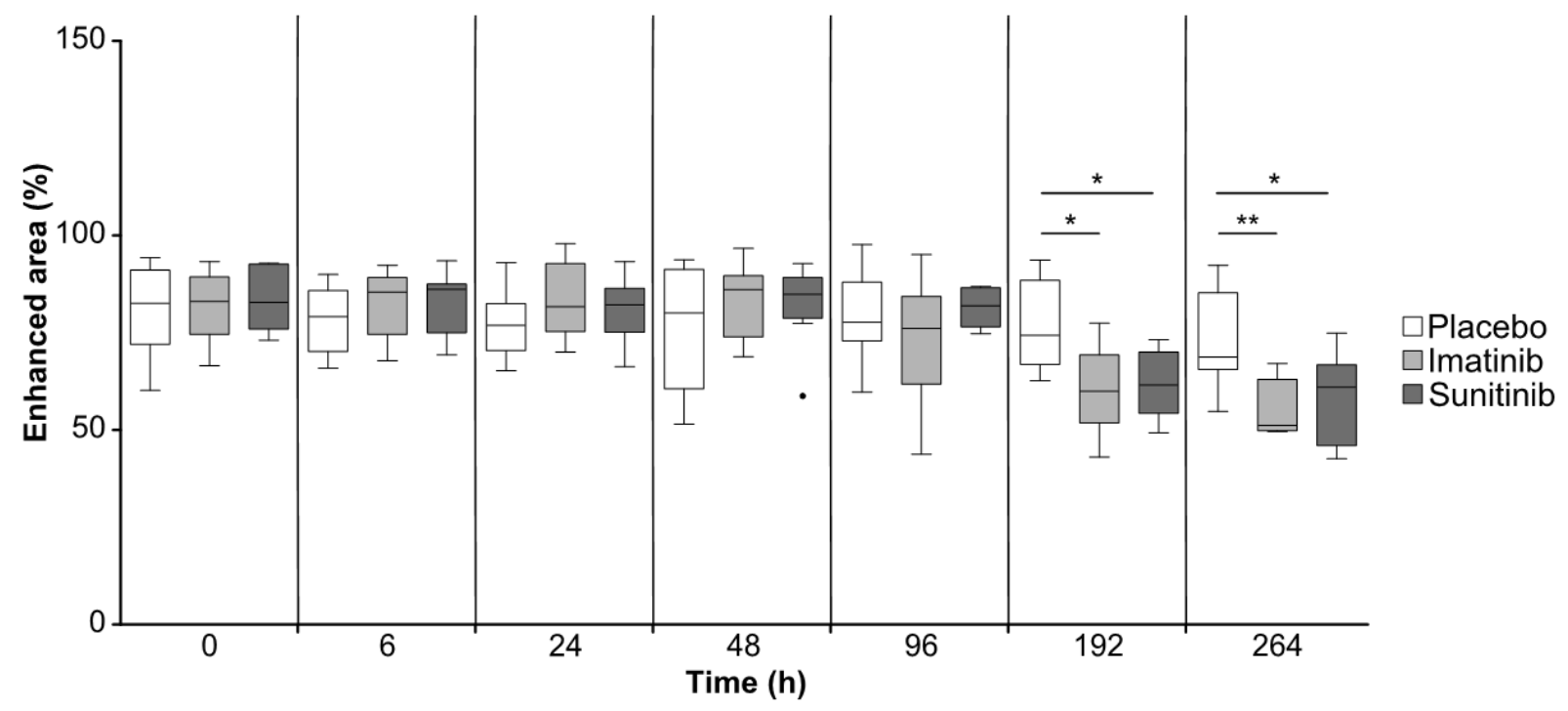

Figure 5 


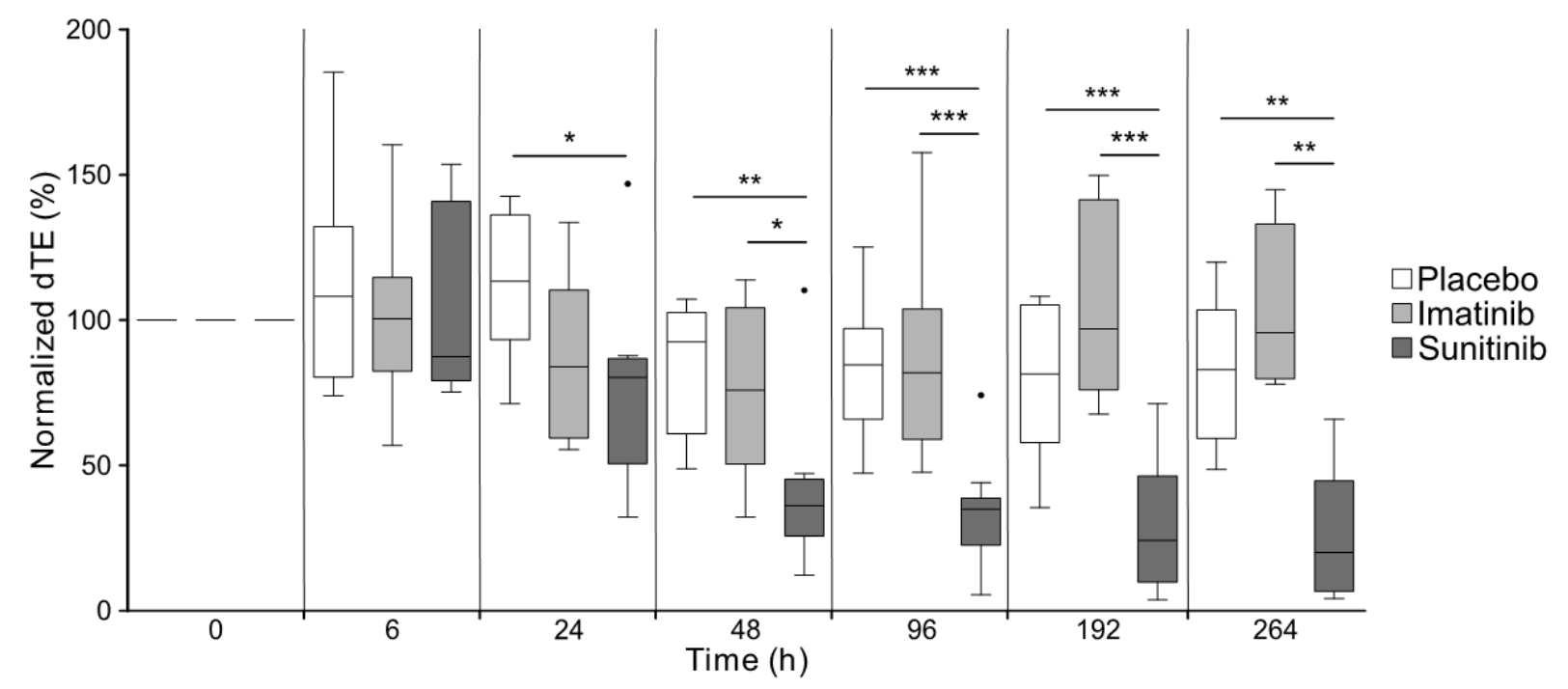

Figure 6

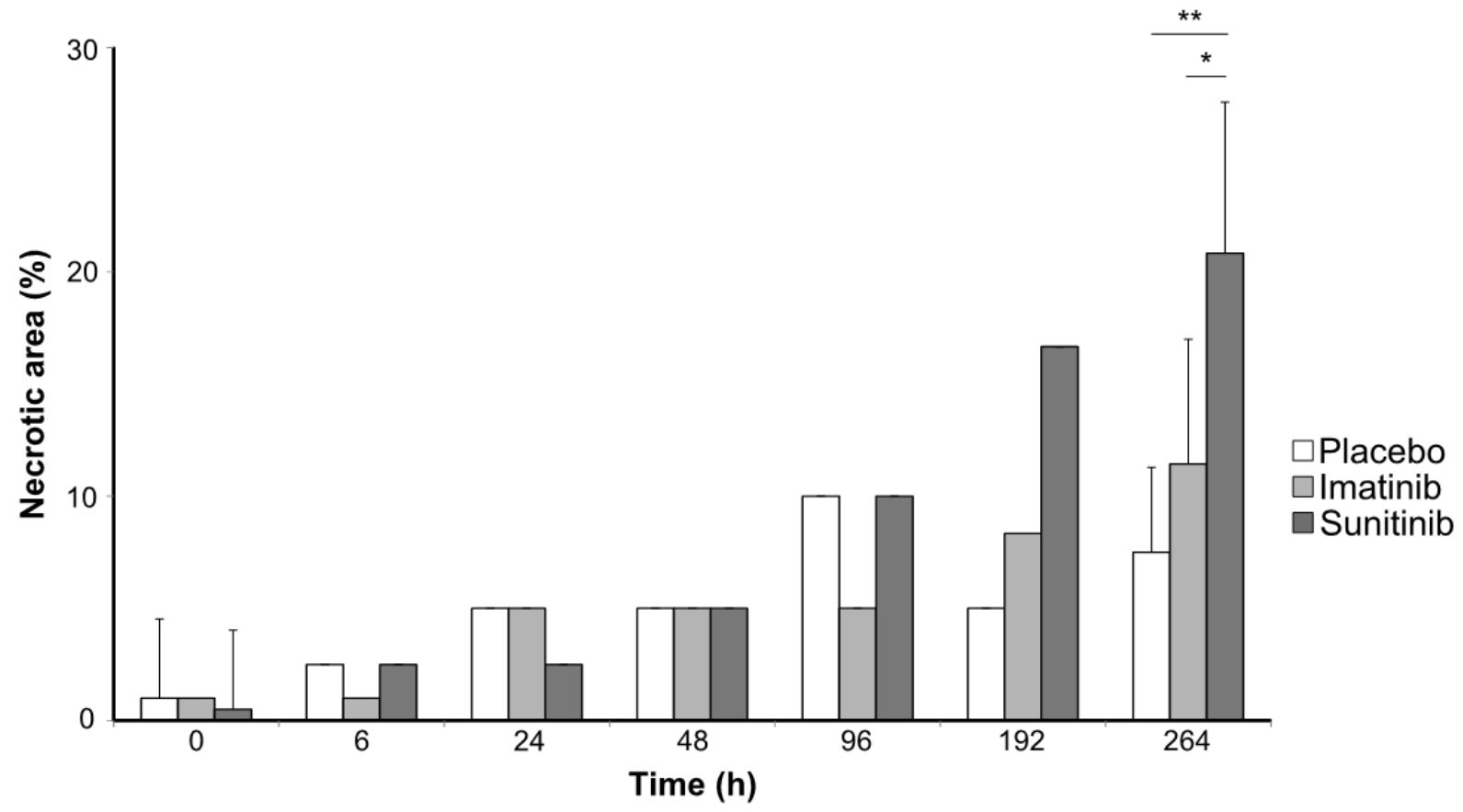

Figure 7 


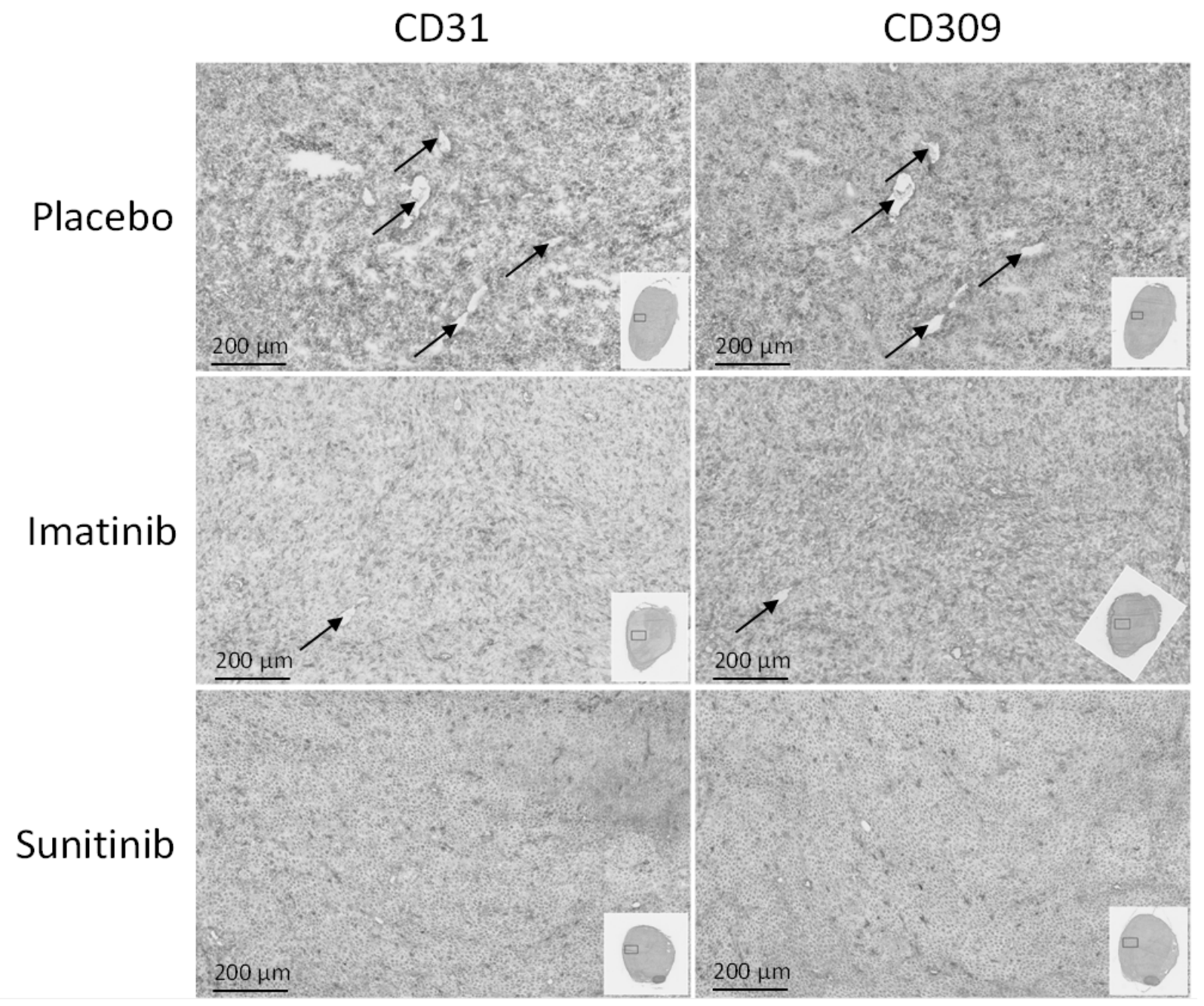

Figure 8 